\title{
Shannon Entropy in Distributed Scientific Calculations on Mobiles Ad-Hoc Networks (MANETs)
}

\author{
Pablo José Iuliano, Luís Marrone \\ LINTI, Facultad de Informatica UNLP, La Plata, Argentina \\ Email: piuliano@info.unlp.edu.ar, lmarrone@linti.unlp.edu.ar
}

Received June 2013

\begin{abstract}
This paper addresses the problem of giving a formal metric to estimate uncertainty at the moment of starting a distributed scientific calculation on clients working over mobile ad-hoc networks (MANETs). Measuring the uncertainty related to the successful completion of a distributed computation on the aforementioned network infrastructure is based on the Dempster-Shafer Theory of Evidence (DST). Shannon Entropy will be the formal mechanism by which the conflict in the scenarios proposed in this paper will be estimated. This paper will begin with a description of the procedure by which connectivity probability is to be obtained and will continue by presenting the mobility model most appropriate for the performed simulations. Finally, simulations will be performed to calculate the Shannon Entropy, after which the corresponding conclusions will be presented.
\end{abstract}

Keywords: MANETs; Shannon; Uncertain; Simulation; Distributed

\section{Introduction}

Mobile computing has been established as the de facto standard for Web access, owing to users preferring it to other connection alternatives. Mobile ad-hoc networks, or MANETs, are currently the focus of attention in mobile computing, as they are the most flexible and adaptable network technology in existence today [1]. These qualities are particularly desirable in the development of applications meant for this kind of infrastructure-a number of American government projects, such as the military investment in resources for the development of this technology, bear witness to this fact.

As previously mentioned, ad-hoc mobile networks are the most flexible and adaptable communication architecture currently in existence. These wireless networks are comprised of interconnected autonomous nodes. They are self-organized and self-generated, which eliminates the need for a centralized infrastructure.

The use of this type of networks as a new alternative for the implementation of distributed computing systems is closely related to the capability to begin calculation, assign parts and collect results once computation is finished. Due to the intrinsic nature of this kind of network, there is no certainty that all the stages involved in this kind of calculation can be completed, which makes estimating the uncertainty in these scenarios, a vital capability.

\section{Measuring the Problem}

The movement patterns of the autonomous nodes, and consequently their interaction, will have a significant impact in the success or failure in collecting the results of a distributed computation. In order to incorporate the notion of connectivity among the nodes, a development will now be presented that shows a formalization of the Connectivity Probability among all the nodes that make up a MANET, that is, the probability that there is a path between one node and any of the rest.

Afterwards, we will take on the task of characterizing the mobility of the nodes, particularly their median speed and direction, the range of their communication signal and the size of the surface on which they circulate. Finally, another section will detail how to estimate Shannon Entropy.

\subsection{Defining Connectivity Probability}

Let $D$ be the domain bounded by the Euclidean plane $R^{2}=\{x, y\}$, within $D$ there are $n$ nodes. At initial time $t=0$, the nodes are somehow located and moving. Let $r_{i}=\left(x_{i}, y_{i}\right)$ be the radius vector of node $i$. Thus, we assume that each node has a communication capacity in the range $r$ : if the distance between two nodes is greater than $r$, then they cannot establish communication. Nodes can transmit information using multihop connections.

Therefore, we can define a network as connected if 
each pair of nodes has a path between them. Connectivity Probability quantifies the likelihood of obtaining a connected network from a set of nodes. Clearly, in scenarios where nodes maintain fixed positions, the connectivity will depend on node density and connection range. Typically the simulations of static scenarios that attempt to determine the connection probability of a number of nodes located randomly in the simulation area introduce a random variable that equals 1 when the network is connected and 0 otherwise. Thus, the average of the said variable over the number of trials gives the Connectivity Probability [2].

For nodes with mobility, time interval divisions are introduced and defined thus:

$$
\tau_{1}^{ \pm}, \tau_{2}^{ \pm}, \tau_{3}^{ \pm}, \ldots
$$

where $\tau_{k}^{+}\left(\tau_{k}^{-}\right)$denotes a time interval during which the network is connected (unconnected). The following function can then be introduced:

$$
f(t)_{+}=\left\{\begin{array}{lll}
1 & \text { si } & t \in \tau_{k}^{+} \\
0 & \text { si } & t \in \tau_{k}^{-}
\end{array}\right.
$$

time intervals can be considered to be randomly distributed, whereby the previously presented function turns into a stochastic process. Consequently, in dynamical environments, Connectivity Probability is defined as follows:

$$
P_{+}=E\left[f(t)_{+}\right]
$$

where $E[$.$] is the expected value, as long as it exists. It$ can be seen that $P_{+}$is time-dependent: $P_{+}=P_{+}(t)$. For stationary stochastic processes $P_{+}=$const . If the stationary process is ergodic, then (3) can be substituted by:

$$
\lim _{\tau \rightarrow \infty} \frac{1}{\tau} \int_{0}^{\tau} f_{+}(t) d t=P_{+}
$$

This equality is equivalent to:

$$
P_{+}=\lim _{\tau \rightarrow \infty} \frac{1}{\tau} \operatorname{mes}\left(T^{+} \bigcap[0, \tau]\right)
$$

where $T^{+}=\bigcup \tau_{k}^{+}$and the mes function are used to measure the total length of the $T^{+} \bigcap[0, \tau]$ interval. The problem of whether the network is connected is thus reduced to determining the existence and estimation of the expected value (3), and if the mobility model is stationary and ergodic, (5) can be used to estimate connectivity [2].

\subsection{Dynamical Systems and Stochastic Processes}

In a homogeneous network system where node capacity and properties are equal among all, it can be reasonably assumed that it can be described by a single system of differential equations, both for a single node and for all of them. If some form of randomness is introduced to node movement, a differential stochastic process will be needed. If, moreover, the stochastic process is considered to be stationary, a system of autonomous differential equations can be used where the right side of the equation does not explicitly depend on time and where nodes differ only from their initial conditions [2]. In dynamical systems theory, a phase flow is defined as a group of changes along the trajectory during a time interval. Dynamical systems are generated by phase flows and can be described by differential equations as follows:

$$
\dot{x}=g(x), x \in \Pi
$$

where $\Pi$ is the phase space, $x$ is a set of coordinates in $\Pi$ (usually position and speed) and the dot indicates that time is the differential. Let $n$ be a number of nodes and $x^{1}, \ldots, x^{n}$ its phase coordinates, then these coordinates satisfy the following differential equation:

$$
\dot{x}^{(k)}=g\left(x^{(k)}\right), k=1, \ldots, n
$$

Thus the dynamic of the $n$ nodes is completely defined by dynamical system (7), which is the direct product of the $n$ copies of the original dynamical system, (6). Its phase space $\hat{\pi} \pi \pi(\pi) \ldots \times={ }^{n}$ is a direct product of the $n$ copies of the initial phase space and phase coordinates $\hat{x}=\left(x^{1}, \ldots, x^{n}\right)$ are a set of coordinates of individual nodes. If system (6) has an invariant measure $\mu$ in $\pi$, system (7) will also have an invariant measure in $\hat{\pi}$ and the direct product will be $\hat{\mu}=\mu_{1} \times \ldots \times \mu_{n}$. In the connectivity problem, phase space $\hat{\pi}$ can be divided into two domains $D$ and $D^{\prime}=\hat{\pi} \backslash D$ thus: when $\hat{x} \in D$, all the nodes out of the existing $n$ can communicate with each other. And when $\hat{x} \in D^{\prime}$, some nodes cannot be reached by some others. Following the approach from dynamical systems, the connectivity probability can be estimated as a time interval when $\hat{x}(t) \in D$.

Estimating the connectivity measure can be significantly simplified if dynamical system (7) is ergodic in $\hat{\pi}$. By definition, a system is ergodic if the measure of some invariant sub domain of the phase space equals zero or the measure of the entire space.

Let $f(\hat{x})$ be a measurable and integrable function in $\hat{\pi}$, for all the solutions of ergodic system (6) there is:

$$
\lim _{T \rightarrow \infty} \frac{1}{T} \int_{0 \pi}^{T} f(\hat{x}(t)) d t=\int_{\text {mes } \hat{\pi}} \frac{f(\hat{x}) d \mu}{,}
$$

where

$$
\text { mes } \hat{\pi}(\tilde{\pi}) \int_{\hat{\pi}} d \mu=\mu
$$

is the measure of the entire phase space [2].

Let $f$ be a function characteristic of a measurable domain $D$ : 


$$
f(\hat{x})=\left\{\begin{array}{lll}
1 & \text { si } & \hat{x} \in D \\
0 & \text { si } & \hat{x} \in D^{\prime}
\end{array}\right.
$$

Since $f$ is limited and $D$ is measurable, $f: \hat{\pi} \rightarrow R$ is integrable. In this case, the left side of (12) is equivalent to the time interval $0 \leq t \leq T$ when $\hat{x}(t)$ resides in the domain $D$.

Thus the Connectivity Probability of an ad-hoc mobile network will be equivalent to the right side of (12):

$$
\frac{\text { mes } D}{\text { mes } \hat{\pi}}
$$

This approximation can be interpreted in terms of the theory of stochastic processes in phase space $\hat{\pi}$. The probability for a system in measurable domain $D$ is determined by formula (11). Let $f(x)$ be a function characteristic of domain $D$ and $x(t)$ the solution of system (6). Thus the function $f(x(t))$ can be interpreted as a stochastic process. Let $E[f(t)]$ be the expected value of the function $f(t)$ at time $t$. If the right side of Equation (6) is not time-dependent, then the stochastic process is stationary. In particular, this means that $E[f(t)]$ does not depend on $t$. If the system is also ergodic, the expected value can be calculated using formula (11):

$$
\lim _{T \rightarrow \infty} \frac{1}{T} \int_{0}^{T} f(\hat{x}(t)) d t=E[f]=\frac{\text { mes } D}{\text { mes } \hat{\pi}}
$$

Therefore, the problem of calculating expected value (3) is reduced to a geometric problem in which we must determine the volume of the domains in a phase space if the process is ergodic [2].

\subsection{Shannon Entropy: Measuring Uncertainty}

Uncertainty, in particular the amount of conflict in the system, will be measured using the Dempster-Shafer Theory of Evidence (DST). Functions for estimating the conflict in a system using a probability distribution must fulfill certain axiomatic requirements [3], namely:

Let $f_{c}$ be the estimator of the amount of conflict and $p=\left\langle p_{1}, p_{2}, \ldots, p_{n}\right\rangle$ the probability distribution, $f_{c}$ must fulfill:

- Expansibility: adding a 0 component to the probability distribution does not modify the value of the uncertainty measure.

- Symmetry: the calculated uncertainty does not vary in relation to the permutation of the arguments.

- Continuity: function $f_{c}$ is continuous for all $p=\left\langle p_{1}, p_{2}, \ldots, p_{n}\right\rangle$.

- Subadditivity: the uncertainty of the joint probability distribution is less than or equal to the uncertainties of the marginal distributions.

- Additivity: for any pair of marginal probability dis- tributions that are non-interactive, the uncertainty of the associated joint distribution must be equal to the sum of the uncertainties of the marginal distributions.

- Monotonicity: uncertainty must increase if the number of elements increases.

- Branching: Let $p=\left\langle p_{1}, p_{2}, \ldots, p_{n}\right\rangle$ over $X=\left\{x_{1}, x_{2}, \ldots, x_{n}\right\}$. If two partitions are generated from $X, A=\left\{x_{1}, \ldots, x_{s}\right\}$ and $B=\left\{x_{s}+1, \ldots, x_{n}\right\}$, then $f_{c}\left(p_{1}, p_{2}, \ldots, p_{n}\right)=$

- Normalization: to ensure uncertainty can be measured in bits, it is required that:

$$
\begin{gathered}
f_{c}\left(p_{A}, p_{B}\right)+f_{c}\left(\frac{p_{1}}{p_{A}}, \frac{p_{2}}{p_{A}}, \ldots, \frac{p_{s}}{p_{A}}\right) \\
+f_{c}\left(\frac{p_{s}+1}{p_{B}}, \frac{p_{s}+2}{p_{B}}, \ldots, \frac{p_{n}}{p_{B}}\right) \\
f_{c}\left(\frac{1}{2}, \frac{1}{2}\right)=1
\end{gathered}
$$

Shannon Entropy will be the formal mechanism by which the conflict will be estimated in this document. This measure of uncertainty stems from a probability distribution obtained from observing the results of an experiment or any other research mechanism. Probability distribution $p$ has the form $p=\langle p(x) \square x \in X\rangle$ where $X$ is the domain of discourse. Additionally, a decreasing function in relation to incidence probability is defined, called anticipatory uncertainty, which must have a decreasing monotonous continuous mapping, and be additive and normalized. This yields that the anticipatory uncertainty of an $x$ result is: $-\log _{2} p(x)$.

Thus, Shannon Entropy, which provides the expected value of the anticipatory uncertainties for each element of the domain of discourse [3], takes the following form:

$$
S(p)=\sum_{x \in X} p(x)=-\sum_{x \in X} p(x) \log g(x)
$$

The normalized version of (14) takes the following form:

$$
S(p)=\frac{1}{\log _{2}(|X|)}-\sum_{x \in X} p(x) \log p(x)
$$

and is the one used to calculate uncertainty in the simulations performed.

\section{Simulation}

Following, we present an adjustment to the previously obtained theoretical results, in order to reach a simulation method that is consistent with them. A description of scenarios posed and results obtained will follow.

\subsection{Adjustment of mes $\hat{\boldsymbol{\pi}}$ and mes $\boldsymbol{D}$}

It is considered that the area where the computational 
model proposed for distributed calculations on ad-hoc mobile networks operate will be small-the work surface will be comparable to that of a university campus, governmental building or office [4]. This results in $\hat{\pi}$ being the total simulation surface and mes $D$, the area where the nodes are in positions that keep the network connected. However, calculating mes $\hat{\pi}$ and mes $D$ as previously proposed is an extremely complicated and laborious task [2]. For this reason, an alternative method is presented to determine first the Connectivity Probability and later the uncertainty involved.

With the goal of validating the scenario put forth in previous sections, the simulation will take place using a modified version of the Monte-Carlo method, where the nodes will be initially located in random positions in such a way that they will form a connected network. Their position will be updated in each instance of the simulation, in accordance with the specifications of the RWMM model [5], and afterwards the network connectivity will be verified. Thus, with the calculation framed within the aforementioned simulation process, the Connectivity Probability will be obtained by means of the $M / N$ quotient, where $N$ is the total number of simulations and $M$ is the number of simulations in which the network was connected where $N$ was great enough. Thus, mes $\hat{\pi}$ is $N$ and mes $D$ is $M$ [2].

\subsection{Results of the Simulation}

Different ad-hoc mobile network topologies will have different Connectivity Probability values, and, therefore, the Shannon Entropy will vary.

In RWMM [5], the nodes for each simulation stage will select a direction in which they will move randomly between $(0,2 \pi]$, and the speed at which they will move will be the expected value uniformly distributed between the speeds of $1 \mathrm{~m} / \mathrm{s}$ and $10 \mathrm{~m} / \mathrm{s}$ - the rates at which we move by foot-which will equal to $3.90 \mathrm{~m} / \mathrm{s}$. When a node reaches the edge of the simulation area, it will rotate 180 degrees and will be placed again within the area, after which the process will continue.

All the simulations begin in a connected network topology and a fixed connectivity radius within which a node can be connected to another. Then, stage after stage, the following operations will take place:

1) For each node node $e_{i}$ of the ad-hoc mobile network, a direction $d i r_{i}$ is randomly chosen between $(0$, $2 \pi$ ], and its position $\operatorname{pos}_{i}=\left(x_{i}, y_{i}\right)$ is updated in accordance with newpos $=\operatorname{pos}_{i}+\left(\operatorname{dir}_{i} \times V \times d t\right)$ where newpos $_{i}$ is the new position of node . $_{i}$.

2) Once all the node positions have been updated, for

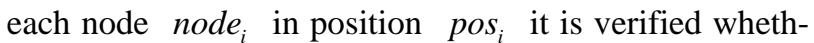
er it can establish a connection with another node node $_{j}$ located within its connection radius. This verification is performed by means of the calculation of the Euclidean distance Dist $_{i, j}$ between the two nodes, later checking whether Dist $_{i, j}<=2 R A D I U S$ is fulfilled, where $R A$ DIUS is the connection radius between the two nodes.

3) If each of the nodes can establish a connection with all other nodes in the network, the resulting network is still connected and the connected topology incidence counter $M$ is increased by one unit.

4) Once the $N$ stages are completed, with $N=10^{5}$ (this number is sufficient to achieve at least 99:999999995\% confidence and a distance between the empirical and the real at most 0.01 using by the Dvoretzky-Kiefer- Wolfowitz inequality [6] and obtain adequate statistical guarantees), Connectivity Probability and Shannon Entropy emerge as a result of the simulation process. Two considerations must be emphasized regarding the calculation of these two measures:

a) As previously mentioned, because the calculation of Connectivity Probability is framed within the simulation process, it can be obtained by means of the $M / N$ quotient, where $N$ is the total number of simulation stages and $M$ is the number of times when the network was connected.

b) Shannon Entropy is calculated by means of the following formula:

$$
=\left\{\begin{array}{ccc}
S(p) & \begin{array}{ccc}
-\left(p \log _{2}(p)+(1-p) \log _{2}(1-p)\right) & \text { si } & 0<p<1 \\
0 & \text { si } & p=0 \vee p=1
\end{array}
\end{array}\right.
$$

As shown, $S(p)$ is zero when $p$ reaches the value zero or one, therefore, there will always be uncertain about the result. Another interesting fact is that the formula of Shannon Entropy which results from this is normalized, since:

$$
\frac{1}{\log _{2}(|X|)} S(p)=\frac{1}{\log _{2}(2)} S(p)=\frac{1}{1} S(p)=S(p)
$$

The results of the simulation scenarios are shown in Figure 1.

\subsection{Correlation between Connectivity Probability and Shannon Entropy}

The theoretical development of the first lines of this document and the results of the simulations will allow the reader to sense the existence of a relationship between Connectivity Probability and uncertainty in a system. For this reason, the following section will analyze this relationship formally. We can begin to study the relationship between Shannon Entropy and Connectivity Probability by observing what happens when the first of the two magnitudes reach its limit values, i.e., its minimum and maximum. The first of these values, equal to zero, is reg- 


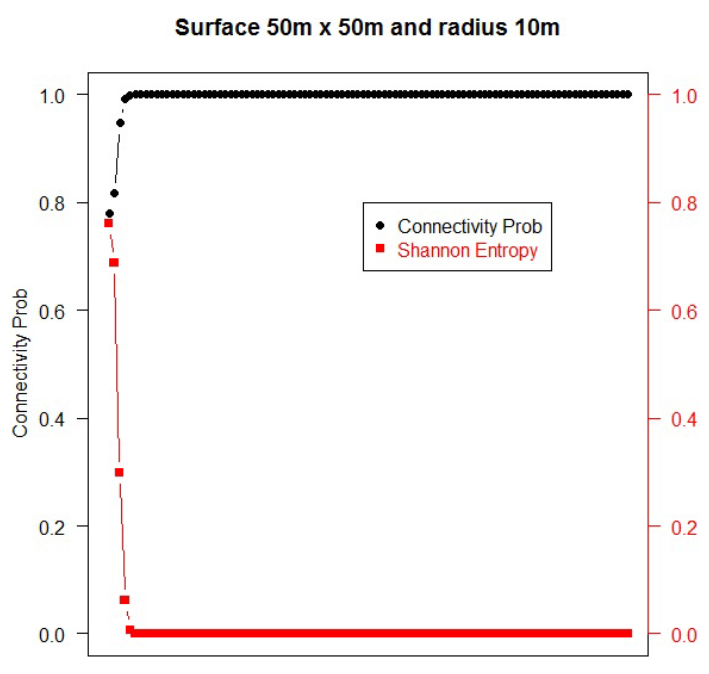

Nodes (amount)

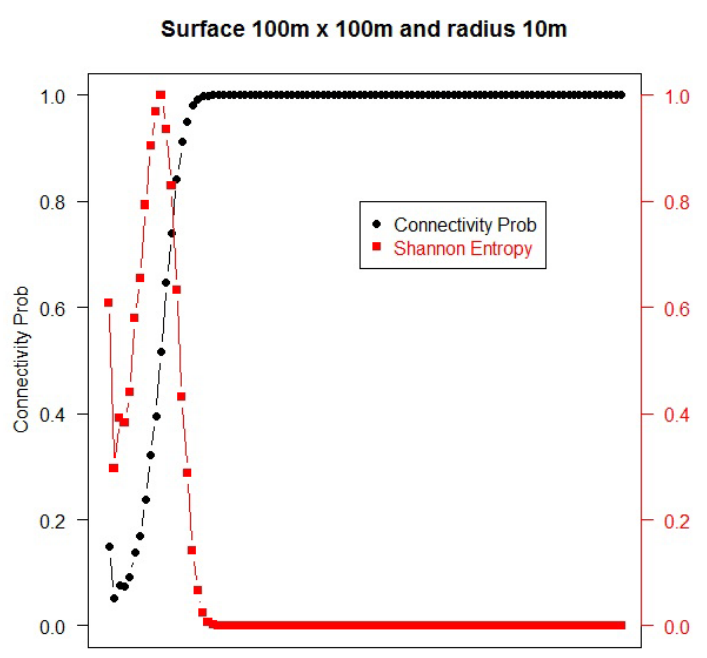

Nodes (amount)

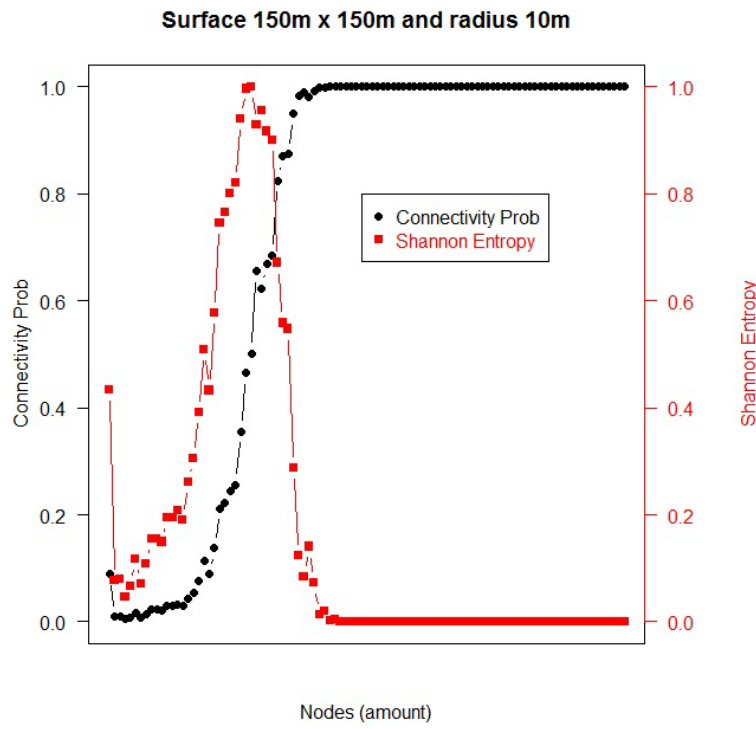

Figure 1. Simulation Results. istered when the Connectivity Probability has reached either one or zero. This shows that when there is no possibility to maintain connectivity or when the possibility is absolute, uncertainty disappears. The maximum value of entropy is reached when the probability takes medium values, which means that there is a state of total uncertainty.

In both cases, the relationship between Shannon Entropy and Connectivity Probability is evident, but for the analysis of the other cases, correlation coefficients must be used.

The correlation coefficient that is most widely used is Pearson's coefficient (r):

$$
\begin{gathered}
\bar{p}=\frac{1}{n} \sum_{i} p_{i} \\
\bar{s}=\frac{1}{n} \sum_{i} s_{i} \\
S_{p}=\frac{1}{n} \sum_{i}\left(p_{i}-\bar{p}\right) \\
S_{s}=\frac{1}{n} \sum_{i}\left(s_{i}-\bar{s}\right) \\
S_{p s}=\frac{1}{n} \sum_{i}\left(p_{i}-\bar{p}\right)\left(s_{i}-\bar{s}\right) \\
r=\frac{S_{p s}}{S_{p} S_{s}}
\end{gathered}
$$

and the extreme values of its possible results are: 0 (no relationship) and \pm 1 (maximal relationship) [7]. The variables analyzed by means of this method, $p$ and $s$, must fulfill certain requirements. The two that are the most relevant to this study are as follows:

1) Variables $p$ and $s$ must be continuous.

2) The relationship between $p$ and $s$ must be linear.

Of the two previous conditions, the most relevant to our observations, or restrictive of them, as the reader prefers, is 2, since it would require the relationship between Connectivity Probability and Shannon Entropy to be linear, which is not the case. Therefore, it is evident that Pearson's Coefficient cannot be used as proposed, since the relationship under analysis is curvilinear. For this reason, this behavior will be analyzed dividing variable $p$ into two segments, which will result in two study groups: the first, we will call $g_{1}$ where $p \in(0,0,5)$, and the second, $g_{2}$, where $p \in[0,5,1)$. Correlation will therefore be calculated in separate groups.

The results obtained and detailed in Table $\mathbf{1}$, show that:

1) $g_{1}$ exhibits direct (positive) dependence between $p$ and $s$, i.e., for large values of $p$ there will be large values of $s$. 
Table 1. Connectivity P/Shannon Entropy Correlation by Groups.

\begin{tabular}{ccccc}
\hline Sup. & N. Grp. 1 & $\begin{array}{c}\text { C. Pearson } \\
\text { Grp. 1 }\end{array}$ & N. Grp 2 & $\begin{array}{c}\text { C. Pearson } \\
\text { Grp 2 }\end{array}$ \\
\hline $50 \mathrm{~m} \times 50 \mathrm{~m}$ & - & - & {$[2,100]$} & -0.993 \\
$100 \mathrm{~m} \times 100 \mathrm{~m}$ & {$[2,11]$} & 0.975 & {$[12,100]$} & -0.965 \\
$150 \mathrm{~m} \times 150 \mathrm{~m}$ & {$[2,28]$} & 0.958 & {$[29,100]$} & -0.971 \\
\hline
\end{tabular}

2) $g_{2}$ shows that the relationship between $p$ and $s$ is inverse (negative) dependence, i.e., for large values $p$ the values of $s$ will be small.

Based on these results, we can conclude the following for MANETs that operate on surfaces of:

1) $50 \mathrm{~m} \times 50 \mathrm{~m}$ : uncertainty will decrease as the amount of network nodes increases, due to greater Connectivity Probability.

2) $100 \mathrm{~m} \times 100 \mathrm{~m}$ : first, if the amount of nodes varies between two and eleven, starting from two and taking eleven as a maximum, as more nodes are added to the network, uncertainty and probability will increase together. Once the twelve node threshold is reached, uncertainty will begin to decrease, while probability increases.

3) $150 \mathrm{~m} \times 150 \mathrm{~m}$ : this case is similar to the last, with the exception that node intervals are displaced-when the amount of nodes varies between two and twentyeight, uncertainty and probability will increase as nodes increase, and if the amount varies between [29, 100], uncertainty will decrease while probability still increases.

It is clear that the most interesting results are those registered in $g_{2}$ for all surfaces, as it is there that computations will have the greatest probability to succeed with less uncertainty. However, the question remains as to what amount of nodes and Connectivity Probability will bring a success certainty high enough to begin computation. One valid criterion is to detect value $v_{i} \in[0.5,1]$ where Connectivity Probability and uncertainty are equal or close enough and operate on the uncertainty interval between $\left[0, v_{i}\right]$. Values $v_{i}$ for the performed simulations are detailed in Table 2. Therefore, for surfaces of $50 \mathrm{~m} \times$ $50 \mathrm{~m}, 100 \mathrm{~m} \times 100 \mathrm{~m}$ and $150 \mathrm{~m} \times 150 \mathrm{~m}$, distributed calculations will begin when 2, 14 and 34 nodes have been reached, respectively.

\section{Conclusion}

The development of this work duly evidenced and documented that the uncertainty existing at the beginning of a distributed computation on a MANET will depend directly on the amount of nodes participating in it and on the surface involved. This statement is based on the results obtained from the simulations detailed in this document, which allowed us to conclude that uncertainty begins to decrease once node density has reached a certain threshold, and that this threshold takes different values
Table 2. Values of $v_{i}$.

\begin{tabular}{cccc}
\hline Sup. & Nodes & Connectivity Probability & Value $\boldsymbol{v}_{\boldsymbol{i}}$ \\
\hline $50 \mathrm{~m} \times 50 \mathrm{~m}$ & 2 & 0.779 & 0.760 \\
$100 \mathrm{~m} \times 100 \mathrm{~m}$ & 14 & 0.738 & 0.828 \\
$150 \mathrm{~m} \times 150 \mathrm{~m}$ & 34 & 0.824 & 0.671 \\
\hline
\end{tabular}

for different surfaces.

Works oriented towards correctly identifying the amount of uncertainty existing at the time the results of a distributed calculation on ad-hoc mobile networks are collected bring the potential benefit that they can be used to develop more intelligent workload distribution strategies that take into account the amount of uncertainty they will have to deal with, which will necessarily results in more efficient computations. In this sense and based on the latest studies oriented towards providing more certain mechanisms as to the conservation of power in the devices that comprise a MANET [8] or on equally relevant studies focusing on achieving the greatest cooperation possible between the nodes of an ad hoc mobile network [9], thus mitigating their intrinsic egotism, the results of having an uncertainty measure that would either indicate that there is no certainty to achieve calculation completion or ensure its success will be twofold. In the first of the aforementioned two fields of study, preventing workload distribution in situations where calculation concretion is not ensured will have a direct repercussion in the conservation of power in devices, which will result in longer operational periods which unable to identify the aforementioned scenarios. The second research field seeks to maximize cooperation among the nodes. With this in mind, in scenarios where completion certainty is medium or low, one possible distribution strategy oriented toward collaboration could be assigning workload only to the most collaborative nodes, to avoid the risk of assigning load to un-collaborative nodes, which, in the event of result collection failure, may take a more selfish or conservationist attitude toward their resources (such as power) and leave the MANET. In a scheme of mobile distributed calculation where all participants offer their collaboration to find the answer to a common interest problem, such as the SETI@Home program [10], measuring uncertainty can be used as a function to grant credit to collaborators - when a participant is notified that there is a medium to high level of uncertainty regarding computation success and they decide to participate nonetheless, more credits can be granted than in scenarios where total certainty of success exists. If more credits mean more benefits for the participant in some way, for example, publicity of the most committed participant in the calculation environment, then we would have a psychological mechanism of positive reinforcement that would promote node collaboration, which would enable a network con- 
formed by more collaborative and satisfied participants that are less egotistic.

All potential strategies of distributed computing over MANETs presented in this document and others that can emerge from an intelligent use of uncertainty measures will bring with them new types of applications that will seize all the power of the underlying network infrastructure.

\section{REFERENCES}

[1] P. Mohapatra and S. Krishhamurthy, "Ad Hoc Networks Technologies and Protocols,” 2004.

[2] T. K. Madsen, F. H. P. Fitzek, R. Prasad and G. Schulte, "Connectivity Probability of Wireless Ad Hoc Networks: Definition, Evaluation, Comparison,” Vol. 35, 2005, ISSN: 0929-6212, pp. 135-151.

[3] C. S. Duque, "Medidas Para Estimar la Incertidumbre Basada en Información Deficiente,” 2012.

[4] F. Bai and A. Helmy, "A Survey of Mobility Models in Wireless Adhoc Networks,” 2008.
[5] C. Bettstetter, H. Hartenstein and X. Perez-Costa, "Stochastic Properties of the Random Waypoint Mobility Model in ACM/Kluwer Wireless Networks," Special Issue on Modeling and Analysis of Mobile Networks, Vol. 10, No. 5, 2004.

[6] F. Xu, "Correlation-Aware Statistical Methods for Sampling-Based Group by Estimates,” Doctoral Thesis, University of Florida, 2009.

[7] P. M. Vallejo, Correlación y Covarianza. Revision, 30 de Octubre de 2007.

[8] S. Prakash, J. P. Saini and S. C. Gupta, "A Vision of Energy Management Schemes in Wireless Ad Hoc Networks,” ISSN: 2278-7844, 2012.

[9] A. E. Hilal and A. B. Mackenzie, "Mitigating the Effect of Mobility on Cooperation in Wireless Ad Hoc Networks," 8th IEEE International Conference on Wireless and Mobile Computing, Networking and Communications (WiMob), 2012.

[10] SETI@home.http://setiathome.berkeley.edu/ 